
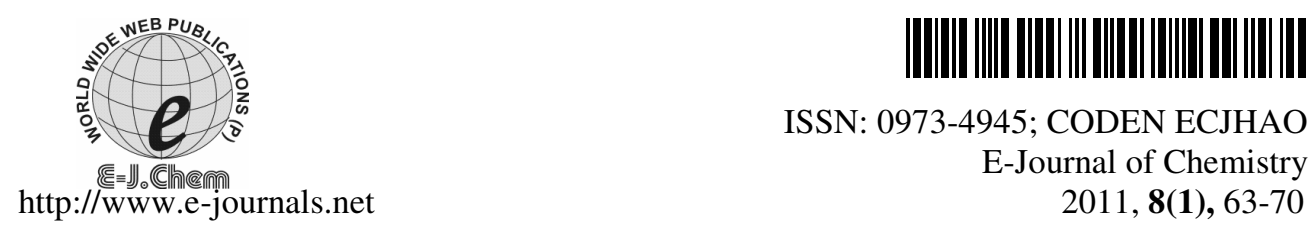

ISSN: 0973-4945; CODEN ECJHAO

E-Journal of Chemistry

2011, 8(1), 63-70

\title{
Studies on Water-Polymer Interactions in the Presence of Aceclofenac at 298.15 K
}

\author{
SITI S.B. AMBOMASE ${ }^{\S}$, SUSHREE TRIPATHY ${ }^{\S}$, \\ MINAKETAN TRIPATHY ${ }^{\S *}$ and UPENDRA N. DASH ${ }^{\#}$ \\ ${ }^{\S}$ Faculty of pharmacy, Campus Puncak Alam \\ Universiti Teknologi MARA (UiTM), Selangore-42300, Malaysia \\ "Department of Chemistry \\ ITER, SOA University, Bhubaneswar-751030, India \\ minaketan@salam.uitm.edu.my
}

Received 20 March 2010; Accepted 15 May 2010

\begin{abstract}
Densities and ultrasonic velocities of aqueous mixtures of methyl cellulose (MC) and polyethylene glycol (PEG) and of methyl cellulose and hydroxyl propyl methyl cellulose (HPMC), over the compositions, 9:1, 8:2 and 6:4, and of MC, HPMC and PEG, over the compositions, 9:0.5:0.5, 8:1:1 and $6: 2: 2$, have been measured with and without acelofenac at $298.15 \mathrm{~K}$ and at atmospheric pressure. The experimental ultrasonic velocities have been used to determine isentropic compressibilities, apparent isentropic molar compressibilities, acoustic impedance, molar compressibility, molar sound velocity, free volume and relative association for the systems with and without aceclofenac. The results have been discussed in terms of solute solvent and solute-solute interactions and various structural effects.
\end{abstract}

Keywords: MC+PEG, MC+HPMC, MC+HPMC+PEG, Aceclofenac, Densities, Ultrasonic velocities

\section{Introduction}

Water-soluble cellulose ether polymers are widely used in food products, adhesives, paints, textiles and paper. These are also used in the pharmaceutical industry ${ }^{1}$. Cellulose ethers have also found widespread use $\mathrm{e}^{2}$ in the formulation of many of the over 4000 pharmaceutical products. In solid dosage forms, they are usually used as tablet binders, film-coating agents, and controlled released matrix formers. Water soluble cellulose ethers, such as MC, HPMC, $\mathrm{HPC}$ and HEC are effective as hydrophilic matrix forming components ${ }^{3,4}$. Cellulose ether polymers have also been used for microencapsulation ${ }^{5}$ and in disperse systems ${ }^{6,7}$, where interfacial interactions are important. The objective of this study was to determine the polymer solvent interactions in presence of the water-insoluble drug, aceclofenac in aqueous 
mixtures of cellulose ether polymers, such as MC, PEG and HPMC, as the drug-polymer interactions can significantly impact the quality of the film coating and the bioavailability of a film coated dosage form. Despite their importance, very little work has been done on drugpolymer interactions ${ }^{8}$. To date, no study has attempted to relate the impact of drugs with aqueous mixtures of two or more polymers. The present investigation has been carried out to study interactions in drug-polymer mixtures at the molecular level by means of density and ultrasonic velocity measurements in the aqueous mixtures of MC and PEG, and of MC and HPMC in the proportions of 9:1, 8:2 and 6:4 and of MC, HPMC and PEG in the 9:0.5:0.5, $8: 1: 1$ and $6: 2: 2$ proportions at $298.15 \mathrm{~K}$ and at atmospheric pressure with and without the drug, aceclofenac.

\section{Experimental}

Methyl cellulose, hydroxyl propyl methyl cellulose 5000 and polyethylene glycol 10,000 were provided by Fluka ${ }^{\circledR}$ Analytical, sigma Aldrich chemie GmbH, Steinbien, Germany. Aceclofenac was provided by Aldrich Chem. Co. Water with a conductivity of $<1.0 \times 10^{-6} \mathrm{~S} \mathrm{~cm}^{-1}$ was obtained by distilling deionized water from alkaline $\mathrm{KMnO}_{4}$ to remove any organic matter. Density values of aqueous mixtures of MC, HPMC and PEG at T(298.15 K) were measured with an Anton paar digital density meter (model DMA 35A) operated in the static mode. The densities have precision better than $\pm 10^{-3} \mathrm{Kg} \mathrm{m}^{-3}$. The ultrasonic velocities of the solutions at $\mathrm{T}(298.15 \mathrm{~K})$ were measured with an accuracy of $\pm 0.5 \mathrm{~ms}^{-1}$ by a single crystal variable path using Multi-frequency Ultrasonic interferometer (Model M-81 F, made by Mittal Enterprises, New Delhi, India) operated at a frequency of $1 \mathrm{MHz}$. In both the cases, temperature was maintained accurate to $\pm 0,05 \mathrm{~K}$. An analytical balance (Model B 303-8, made by METTLERTOLEDO (m) SDN, BHD) accurate to $0.1 \mathrm{mg}$ was used for weight measurements.

\section{Procedure}

\section{Preparation of aqueous solution of methyl cellulose bases}

Aqueous solutions of MC, HPMC and PEG were prepared on a w/v\% (1\%). Using distilled water as a solvent $\mathrm{MC}(0.9 \%, 0.8 \%$ and $0.6 \%)$ was dispersed in the distilled water together with PEG $(0.1 \%, 0.2 \%$ and $0.4 \%)$ respectively, at $298.15 \mathrm{~K}$. Another three stock solutions were prepared using HPMC $(0.1 \%, 0.2 \%$ and $0.4 \%)$ together with $\mathrm{MC}(0.9 \%, 0.8 \%$ and $0.6 \%)$. Similarly MC $(0.9 \%, 0.8 \%$ and $0.6 \%)$ was dispersed in water together with HPMC $(0.05 \%, 0.1 \%$ and $0.2 \%)$ and PEG $(0.05 \%, 0.1 \%$ and $0.2 \%)$ respectively at $298.15 \mathrm{~K}$. The preparation of aqueous mixture of the polymers in the above composition was done, by using a mechanical stirrer (Homogenizer CAT, model X120 made by M. Zipper GmbH, Germany with a 4 blade propeller, f $60 \mathrm{~mm}$.) at $1000 \mathrm{rpm}$ in a batch size of $200 \mathrm{~mL}$ for at least $1 \mathrm{~h}$ with continuous stirring to assure a complete solubilisation. These nine stock solution thus prepared were labeled as S1, S2, S3, S4, S5, S6, S7, S8 and S9 respectively and stored at $4{ }^{\circ} \mathrm{C}$ until required and subjected directly to density and ultrasonic velocity measurement at $298.15 \mathrm{~K}$.

\section{Preparation of aqueous solutions of MC bases with the drug aceclofenac}

MC $(0.9 \%, 0.8 \%$ and $0.6 \%)$ was dispersed in distilled water containing aceclofenac $(0.5 \mathrm{~g})$ and PEG $(0.9 \%, 0.8 \%$ and $0.6 \%)$ at 298.15 K. Similarly mixture of MC and HPMC and of MC, HPMC \& PEG with aceclofenac were obtained and the homogenous solutions were prepared as above using a mechanical shaker and stored at $4{ }^{\circ} \mathrm{C}$ until required for the density and ultrasonic velocity measurement at $298.15 \mathrm{~K}$. The stock solutions were labeled as Q1, Q2, Q3, Q4, Q5, Q6, Q7, Q8 and Q9 respectively (Table 1). 
Table 1. Composition of the aqueous polymer mixture with and without drug (aceclofenac)

\begin{tabular}{clcc}
\hline Sample & \multicolumn{1}{c}{ Components } & $\begin{array}{c}\text { Weight, in } \mathrm{g} \text { in } 200 \mathrm{~mL} \text { of } \\
\text { distilled water }\end{array}$ & $\begin{array}{c}\text { \% ratio of polymers } \\
\text { in the mixture. }\end{array}$ \\
\hline S1 & MC + PEG & $1.8+0.2$ & $9: 1$ \\
S2 & MC + PEG & $1.6+0.4$ & $8: 2$ \\
S3 & MC + PEG & $1.2+0.8$ & $6: 4$ \\
Q1 & MC + PEG + Aceclofenac & $1.8+0.2+0.5$ & $9: 1$ \\
Q2 & MC + PEG + Aceclofenac & $1.6+0.4+0.5$ & $8: 2$ \\
Q3 & MC + PEG + Aceclofenac & $1.2+0.8+0.5$ & $6: 4$ \\
S4 & MC + HPMC & $1.8+0.2$ & $9: 1$ \\
S5 & MC + HPMC & $1.6+0.4$ & $8: 2$ \\
S6 & MC + HPMC & $1.2+0.8$ & $6: 4$ \\
Q4 & MC + HPMC + Aceclofenac & $1.8+0.2+0.5$ & $9: 1$ \\
Q5 & MC + HPMC + Aceclofenac & $1.6+0.4+0.5$ & $8: 2$ \\
Q6 & MC + HPMC + Aceclofenac & $1.2+0.8+0.5$ & $6: 4$ \\
S7 & MC + HPMC + PEG & $1.8+0.1+0.1$ & $9: 0.5: 0.5$ \\
S8 & MC + HPMC + PEG & $1.6+0.2+0.2$ & $8: 1: 1$ \\
S9 & MC + HPMC + PEG & $1.2+0.4+0.4$ & $6: 2: 2$ \\
Q7 & MC + PEG + HPMC + & $1.8+0.1+0.1+0.5$ & $9: 0.5: 0.5$ \\
& Aceclofenac & & $8: 1: 1$ \\
Q8 & MC + PEG + HPMC + & $1.6+0.2+0.2+0.5$ & $6: 2: 2$ \\
& Aceclofenac & & \\
Q9 & MC + PEG + HPMC + & $1.2+0.4+0.4+0.5$ & \\
\hline
\end{tabular}

\section{Results and Discussion}

The densities and ultrasonic velocity values of water ${ }^{9}$ and of various samples (S1 to S9 without drug and Q1 to Q9 with drug) measured at $298.15 \mathrm{~K}$ are shown in Figure 1 and 2, respectively. The values of density and sound velocity displayed in figures represent the average of at least three independent measurements. As observed, the density value of aqueous mixture of MC and PEG (9:1) is less than that of water and increase when PEG proportion increases (i.e., in 8:2 mixture) and then becomes less in 6:4 ratio mixture. The lowering of density value with the addition of MC and PEG to water may be due to the fact that water molecules because of the smaller size, as compared to the polymer materials causing the latter to swell resulting in the increased volume and hence the density value decreases. The higher density value of 8:2 ratio MC and PEG mixture than water and also than $(9: 1)$ ratio mixture may be due to the fact that the dissolution of polymer in water is a slow process and occurs in two stages ${ }^{10}$; First the solvent, water molecules penetrate deep into the polymer to produce a swollen gel, and secondly the strong polymer-solvent (water) interactions overcoming the polymer - polymer intermolecular force of interaction transform the swollen gel into true solution resulting in a decrease in volume thereby increasing the density. Further, as the PEG proportion increases in aqueous MC mixture (i.e., 6:4 ratioMC-PEG mixture), PEG exists in fully swollen state thereby causing an increase volume and hence a decrease in density.

As can be seen from Figure 1, the density values for the aqueous mixture of $\mathrm{MC}$ and HPMC (S4, S5 and S6) are lower than those of water and also of MC + PEG mixture and tend to decrease as the proportion of HPMC increases in the mixture. Similarly, the density 
values of the samples (S7, S8 and S9) containing all the three polymers are found to be lower than that of water and of the mixture of MC and PEG and tend to increase with increase in the proportion of HPMC and PEG. The decrease and increase in density values with respect to that of water may thus be accompanied with the formation of the swollen gel and its transformation into the true solution, respectively.

The variation of the density values of the aqueous mixture of the polymers containing the drug, aceclofenac (samples, Q1 to Q9) is also displayed in Figure 1. As can be seen, the density values of the aqueous mixture of polymers with drug are less than that of water but more than those of the mixtures without drug. However the density values show maxima in the samples Q2 \& Q5 (i.e., in 8:2 ratio mixtures of MC and PEG and MC and HPMC) and increase continuously the mixtures containing all the three polymers. As usual, the formation of the swollen gel and the transformation of the swollen gel into true solution may be the plausible cause of the decreased and the increased density values of the samples, respectively.

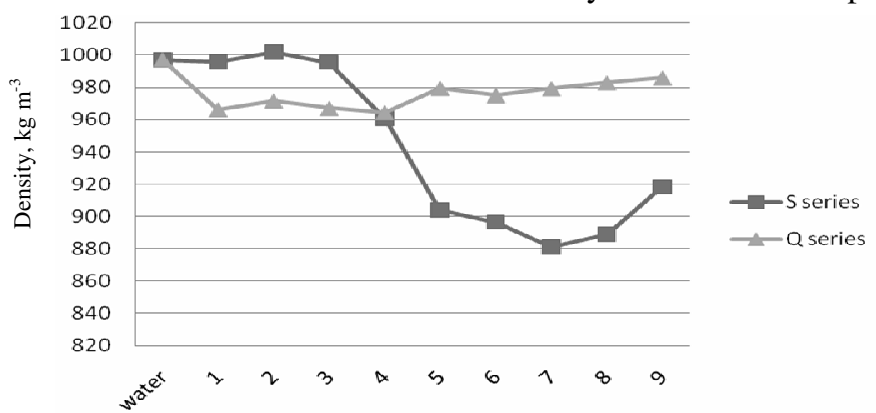

Figure 1. Shows the density $\left(\mathrm{Kg} \mathrm{m}^{-3}\right)$ of the different polymeric solutions in presence and absence of the drug

As expected, the aqueous mixture of the different combinations of MC\& PEG, MC\& HPMC and MC, HPMC and PEG and also their mixtures containing aceclofenac have the higher values of sound velocity than water (Figure 2). As can be seen, the aqueous mixtures of polymers containing aceclofenac have comparatively low values of sound velocity as compared to their counter parts without aceclofenac. The lowering of sound velocity in the presence of the drug may be attributed to the decrease in the number of molecules because of inclusion complex formation of the drug molecules are taken into the hydrophobic central cavity of the water soluble polymer molecules resulting in the decrease of number of molecules in the system and hence the lower sound velocity. This is consistent with the result obtained from the lower density values of the polymer mixtures containing the drug aceclofenac.

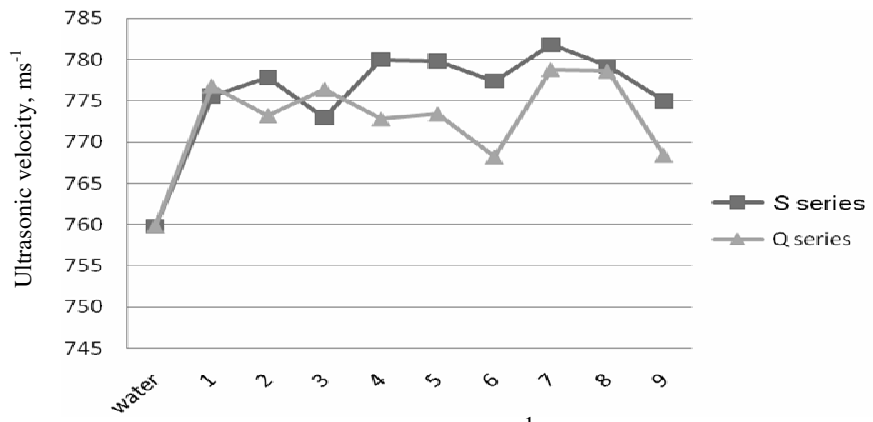

Figure 2. Showing the ultrasonic velocities $\left(\mathrm{ms}^{-1}\right)$ of the different polymeric solutions in presence and absence of the drug acelofenac 
From the results of the sound velocity (U) and density (d), the isentropic compressibility, $\mathrm{K}_{\mathrm{s}}$ of the samples was calculated ${ }^{9}$ as

and the internal pressure ${ }^{11}$ as

$$
\mathrm{K}_{\mathrm{s}}=1 / \mathrm{dU}^{2}
$$

$$
\pi_{\mathrm{i}}=\left(\mathrm{K}_{\mathrm{s}}^{0}-\mathrm{K}_{\mathrm{s}}\right)
$$

Where, $\mathrm{K}_{\mathrm{s}}{ }^{0}$ is the isentropic compressibility of water. The apparent isentropic molar compressibility $\mathrm{K}_{\mathrm{s}}{ }^{0}$ of the samples have been computed from Equation ${ }^{9}$

$$
\mathrm{K}_{\mathrm{s}}^{0}=1000 \mathrm{~K}_{\mathrm{s}} \mathrm{c}^{-1}-\mathrm{K}_{\mathrm{s}}^{0} \mathrm{~d}_{0}^{-1}\left(1000 \mathrm{~d} \mathrm{c}^{-1}-\mathrm{M}\right)
$$

Where, $\mathrm{K}_{\mathrm{s}}^{0}$ and $\mathrm{d}_{0}$ are the isentropic compressibility and density respectively of water at $298.15 \mathrm{~K}$. The values of density and sound velocity enable us to estimate the magnitude of relative association from the relation ${ }^{12}$.

$$
\mathrm{R}_{\mathrm{o}}=\mathrm{d} / \mathrm{d}_{\mathrm{o}}\left(\mathrm{U}_{\mathrm{o}} / \mathrm{U}\right)^{1 / 3}
$$

Where, $U_{o}$ is the sound velocity of water at $298.15 \mathrm{~K}$. The acoustic impedance $\mathrm{Z}$ has been calculated from the relation ${ }^{13}$.

$$
\mathrm{Z}=\mathrm{Ud}
$$

The values of the $K_{s}, R_{o}, Z$ and $K_{s}{ }^{\circ}$ of the samples are given in Table 2 and 3 respectively. The value of free volume, $V_{f}$ has been calculated from the relation ${ }^{13}$.

$$
\mathrm{V}_{\mathrm{f}}=\mathrm{V}_{\mathrm{m}}-\mathrm{b}
$$

Where $\mathrm{V}_{\mathrm{m}}$ is the molar volume of the mixture and is given by

$$
\begin{aligned}
\mathrm{V}_{\mathrm{m}} & =\mathrm{M}_{\mathrm{av}} / \mathrm{d} \quad \text { and } \\
\mathrm{M}_{\mathrm{av}} & =\sum \mathrm{n}^{\mathrm{i}} \mathrm{m}^{\mathrm{i}} / \sum \mathrm{n}^{\mathrm{i}}
\end{aligned}
$$

Table 2. Values of isentropic compressibility $\left(\mathrm{K}_{\mathrm{s}}\right)$, acoustic impedance $(\mathrm{Z})$ and relative association $\left(R_{0}\right)$ of polymeric samples in presence and absence of the drug aceclofenac

\begin{tabular}{cccc}
\hline Sample & $10^{9} \mathrm{~K}_{\mathrm{s}}\left(\mathrm{Pa}^{-1}\right)$ & $\mathrm{Z}, \mathrm{kg} \mathrm{m}^{-2} \mathrm{~s}^{-1}$ & $\mathrm{R}_{\mathrm{o}}$ \\
\hline water & 1.73734 & 757558.6 & 1 \\
S1 & 1.66945 & 772303.7 & 0.99186 \\
S2 & 1.64991 & 779238.9 & 0.99700 \\
S3 & 1.68121 & 769482.9 & 0.99268 \\
S4 & 1.71098 & 749307 & 0.95510 \\
S5 & 1.81944 & 704822.2 & 0.89870 \\
S6 & 1.84601 & 696822.5 & 0.89216 \\
S7 & 1.85698 & 688804.9 & 0.87528 \\
S8 & 1.85299 & 692591.9 & 0.88401 \\
S9 & 1.81316 & 711643.8 & 0.91490 \\
Q1 & 1.71493 & 750660.7 & 0.96208 \\
Q2 & 1.72114 & 751434.4 & 0.96906 \\
Q3 & 1.71528 & 750895.3 & 0.96304 \\
Q4 & 1.73597 & 745404.2 & 0.96194 \\
Q5 & 1.70691 & 757506.6 & 0.97655 \\
Q6 & 1.73808 & 748956.6 & 0.97425 \\
Q7 & 1.68366 & 762639.9 & 0.97409 \\
Q8 & 1.67836 & 765247 & 0.97775 \\
Q9 & 1.71797 & 757527.1 & 0.98506 \\
\hline
\end{tabular}


Table 3. Values of apparent isentropic molar compressibility $\left(\mathrm{K}_{\mathrm{s}}{ }^{\circ}\right)$ of polymeric aqueous solution in the presence of aceclofenac

\begin{tabular}{ccc}
\hline Sample /polymer & $\mathrm{K}_{\mathrm{s}}{ }^{0}$ Polymers, $\mathrm{m}^{3} \mathrm{~mol}^{-1} \mathrm{pa}^{-1}$ & $\mathrm{~K}_{\mathrm{s}}{ }^{0}$ Aceclofenac, $\mathrm{m}^{3} \mathrm{~mol}^{-1} \mathrm{pa}^{-1}$ \\
\hline Q1.MC & -0.00019 & -0.00067 \\
Q1.PEG & -0.00168 & -0.00067 \\
Q2.MC & -0.00021 & -0.00068 \\
Q2.PEG & -0.00085 & -0.00068 \\
Q3.MC & -0.00028 & -0.00067 \\
Q3.PEG & -0.00042 & -0.00067 \\
Q4.MC & -0.00019 & -0.00067 \\
Q4.HPMC & -0.00168 & -0.00067 \\
Q5.MC & -0.00021 & -0.00068 \\
Q5.HPMC & -0.00085 & -0.00068 \\
Q6.MC & -0.00028 & -0.00068 \\
Q6.HPMC & -0.00042 & -0.00068 \\
Q7.MC & -0.00019 & -0.00068 \\
Q7.PEG & -0.00341 & -0.00068 \\
Q7.HPMC & -0.00341 & -0.00068 \\
Q8.MC & -0.00021 & -0.00069 \\
Q8.PEG & -0.00171 & -0.00069 \\
Q8.HPMC & -0.00171 & -0.00069 \\
Q9.MC & -0.00029 & -0.00069 \\
Q9.PEG & -0.00086 & -0.00069 \\
Q9.HPMC & -0.00086 & -0.00069 \\
\hline
\end{tabular}

Where $\mathrm{n}^{\mathrm{i}}$ is the number of moles and $\mathrm{m}^{\mathrm{i}}$ is the molecular mass of the $i^{\text {th }}$ component in the mixture and ' $\mathrm{d}$ ' is the density of the corresponding aqueous mixtures of the polymers. In equation (6), ' $b$ ' is the Vander walls constant and obtained from the relation ${ }^{14}$.

$$
\mathrm{b}=\mathrm{M}_{\mathrm{av}} / \mathrm{d}\left[1-\left(\mathrm{RT} / \mathrm{M}_{\mathrm{av}} \mathrm{U}^{2}\right)\left[\left(1+\left(\mathrm{M}_{\mathrm{av}} \mathrm{U}^{2} / 3 \mathrm{RT}\right)^{1 / 2}-1\right]\right.\right.
$$

Where $\mathrm{R}$ is the universal gas constant, $\mathrm{T}$ (298.15) $\mathrm{K}$ and $\mathrm{M}_{\mathrm{av}}$ is the average molecular mass of the mixture as given in equation (8). The value of $V_{f}$ along with that of internal pressure, $\pi_{\mathrm{i}}$ are given in Table 4 .

Table 4. Values of internal pressure $\left(\pi_{\mathrm{i}}\right)$ and the free volume $\left(\mathrm{V}_{\mathrm{f}}\right)$ of the polymeric samples in presence and absence of the drug aceclofenac

\begin{tabular}{ccc}
\hline Sample & $\pi_{\mathrm{i}}(\mathrm{Pa})$ & $\mathrm{V}_{\mathrm{f}}, \mathrm{m}^{3} \mathrm{~mol}^{-1}$ \\
\hline S1 & -713.4977017 & -303.697 \\
S2 & -816.4010381 & -256.2 \\
S3 & -1110.929312 & -171.384 \\
S4 & -602.9702042 & -374.52 \\
S5 & -559.6195515 & -405.06 \\
S6 & -540.7383638 & -421.598 \\
S7 & -588.7715738 & -377.207 \\
S8 & -626.2133166 & -348.641 \\
S9 & -724.091101 & -290.999 \\
Q1 & -693.3946759 & -312.654 \\
Q2 & -794.9584757 & -261.93 \\
Q3 & -1079.329874 & -176.848 \\
Q4 & -608.5281462 & -368.584 \\
Q5 & -609.337612 & -369.743 \\
Q6 & -591.7161734 & -381.986 \\
Q7 & -656.5275665 & -337.216 \\
Q8 & -693.9497517 & -314.274 \\
Q9 & -781.05786 & -268.089 \\
\hline
\end{tabular}


As can be seen from Table 2, the values of isentropic compressibility, $\mathrm{K}_{\mathrm{s}}$ show a minima in the 8:2 ratio of $\mathrm{MC}$ and PEG mixture while there is an increasing trend shown by the MC and HPMC mixture and a decreasing trend by the MC, HPMC and PEG mixtures. However, the mixture of the polymers containing aceclofenac show isentropic compressibility maxima at 8:2 ratio of MC \& $\mathrm{PEG}$ mixture and minima at the similar combination of MC and HPMC and 8:1:1 ratio combination of MC, HPMC and PEG. Addition of polymers to water results in the breaking of the $3 d$-polymeric structure of water and formation of some complex structure/aggregate. The tendency of aggregate formation increases in the presence of the drug, aceclofenac as a result of which the $\mathrm{K}_{\mathrm{s}}$ values become comparatively smaller than the values of the samples containing no aceclofenac. It means that the addition of the drug to the aqueous mixtures of polymers of different compositions makes the medium electrostrictive as a result of which the isentropic compressibility decreases, and the internal pressure increases.

It is observed that the apparent isentropic molar compressibility, $\mathrm{K}_{\mathrm{s}}{ }^{\mathrm{o}}$ decrease with addition of drug to the aqueous mixtures of the polymers. The values of $\mathrm{K}_{\mathrm{s}}{ }_{\mathrm{s}}$ are negative and this negative value can be explained in terms of two different phenomena; electrostriction and hydrophobic salvation. It means that the medium is compressed in presence of drug, and does not respond to further application of pressure due to hydrophobic salvation due to the fact that the drug molecules enter into the hydrophobic central cavity of the water soluble polymer molecules.

A perusal of Table 2 shows that the acoustic impendence $\mathrm{Z}$ is higher for the mixture of MC\& PEG and is less for the mixtures of MC and HPMC and of MC, HPMC and PEG as compared with that of water. The $\mathrm{Z}$ value decreases for some mixture with addition of the drug. Since the acoustic impedance is a measure of the resistance offered by the liquid medium to the sound wave and is a function of the elastic properties of the medium, the variations of $\mathrm{Z}$ values suggest that probably in certain composition, the medium starts losing its elastic property.

The value of the relative association, $\mathrm{R}_{\mathrm{o}}$ (Table 2) show a slight decrease from unity as we move from pure water to aqueous mixtures containing $\mathrm{MC}$ and PEG but show a significant decrease for the mixtures of MC and HPMC and of MC, HPMC and PEG. The decrease in $R_{0}$ values is also marked in the mixtures of the polymers containing aceclofenac. The relative association is influenced by two factors; (i) breaking of the associated solvent molecules on addition of solute to it and (ii) the solvation of the solute molecules (here polymers and the drug).The former results in the decrease and the latter, in the increase of $R_{o}$. In the present study, $R_{o}$ decreases suggesting to the view that the water structure is disrupted and then the water molecules as stated earlier, penetrate deep into the polymer molecules giving rise to swollen gel and the hydrophobic solvation of the drug molecules.

Internal pressure $\pi_{\mathrm{i}}$ plays an important role in elucidating molecular interactions as this represents the resultant of the forces of attraction and repulsion between the molecules. The free volumes $V_{\mathrm{f}}$ is the effective volume accessible to the center of a molecule in a liquid. The structure of a liquid is determined by strong repulsive forces in the liquid with the relatively weak attractive forces providing internal pressure which holds the liquid molecules together. The free volume seems to be conditional by the repulsive forces while the internal pressure is more sensitive to attractive forces. These two factors together uniquely determine the entropy (i.e., disorderness) of the system. The internal pressure, free volume and temperature are the thermodynamic variables that describe the liquid system of fixed composition. As observed, the internal pressure and free volume are negative and vary 
in opposite direction for the aqueous mixtures of polymers with and without the drug aceclofenac. The negative value of these two parameters indicate that there is a decrease in cohesive forces leading to the disruption of water structure in the systems containing the mixtures of polymer molecules and the drug as a result of which water molecules are entrapped in voids formed during uncoiling of polymeric chains in solution giving rise to swollen gel and the hydrophobic solvation of the drug.

\section{Conclusion}

In this paper, densities and ultrasonic velocities of aqueous mixtures of MC \& PEG and MC \& HPMC in the proportions of 9:1, 8:2 and 6:4 and of MC, HPMC \& PEG in the proportions of 9:0.5:0.5, 8:1:1 and 6:2:2 have been measured with and without the drug aceclofenac at $298.15 \mathrm{~K}$ and comparison with the data available in the literature has been made for water. The measured density values of the aqueous mixture of the polymers with $\&$ without aceclofenac were found to be lower as compared to that of water but the densities of the polymer mixture containing aceclofenac are not so much affected as those without the drug. The lower density values of the polymer mixtures as compared with water may be attributed to the formation of the swollen state in aqueous polymers resulting in the increased volume and the higher density values may be due to the transformation of the swollen gel into true solutions entailing a decrease in volume.

The ultrasonic velocities of the polymer systems with and without the drug are higher than that of water. However, the ultrasonic velocities of the mixtures, containing the drug are comparatively lower than that of their counter parts. The lowering of sound velocity may be due to the decrease in the number of molecules because of inclusion complex formation of the drug molecules with the polymer molecules.

From the densities and ultrasonic velocities, the acoustic parameters such as isentropic compressibility, apparent molar isentropic compressibility, relative association, acoustic impedance, internal pressure and free volume of the systems have been calculated and discussed in the light of rupture of water structure leading to the formation of swollen gel in polymer mixtures and hydrophobic solvation of the drug with water soluble polymer molecules.

\section{References}

1. Wade A and Weller P J, (Eds)., Handbook of Pharmaceutical Excipients, American Pharmaceutical Association, Washington, D.C., 1994.

2. Physician's Desk Reference, Medical Economies Co., Oradell, NJ, 1998.

3. Alderman D A, Int J Pharm Technol Prod Mfr., 1984, 5, 1-9.

4. Salsa T, Veiga F and Pina M E, Drug Dev Ind Pharm., 1997, 23(9), 929-938.

5. Conti B, Giunchedi P and Conte U, STP Pharma Sci., 1997, 7, 331-342.

6. Daniels R and Barta A, Eur J Pharm Bio Pharm., 1994, 40, 128-133.

7. Duro R, Souto C, Gomez-Amoza J L, Martinez-Pacheco R and Concheiro A, Drug Dev Ind Pharm., 1999, 25(7), 817-829.

8. Nair R, Nyamweya N, Gonen S, Martinez - Mieanda L J and Hoag S W, Int J Pharm., 2001, 225, 83-96.

9. Dash U N and Supkar S, Acoustic lett., 1992, 16, 135.

10. Dash U N, Textbook of Biophysical Chemistry, Macmillan India Ltd., 2006.

11. Srivastava S P,Prakash S and Prakash O, Z Physik Chem., (Germany) 1966, 50, 11.

12. Dash U N, Roy G S and Mohanty S, Indian J Chem., 2002, 41A, 2507.

13. Dash U N, Roy G S and Mohanty S, Ultra Sci Phys Sci., 2003, 15(1), 1-15.

14. Dhanalakshmi A, Jasmine E and Rani V, J Acoust Soc India., 1994, 22, 1. 


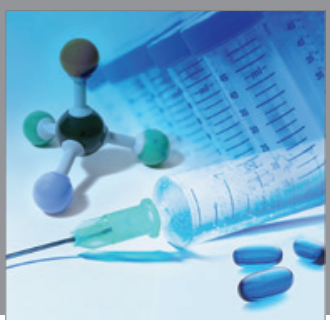

International Journal of

Medicinal Chemistry

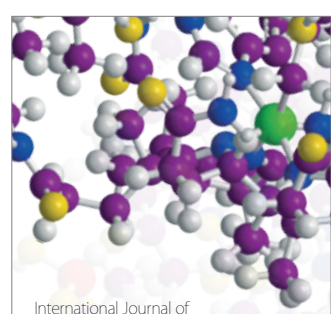

Carbohydrate Chemistry

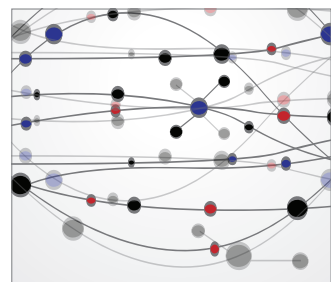

The Scientific World Journal
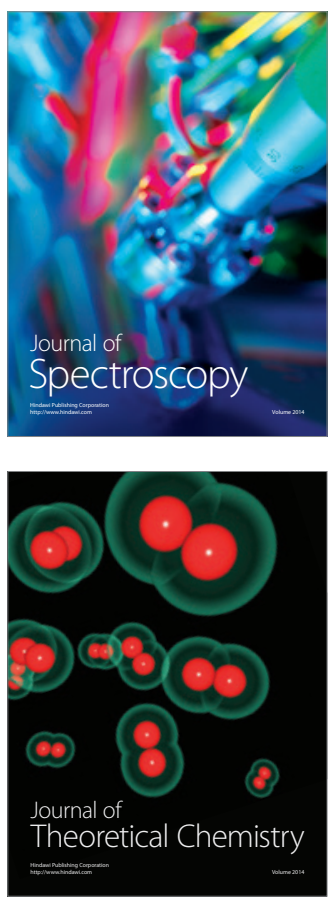
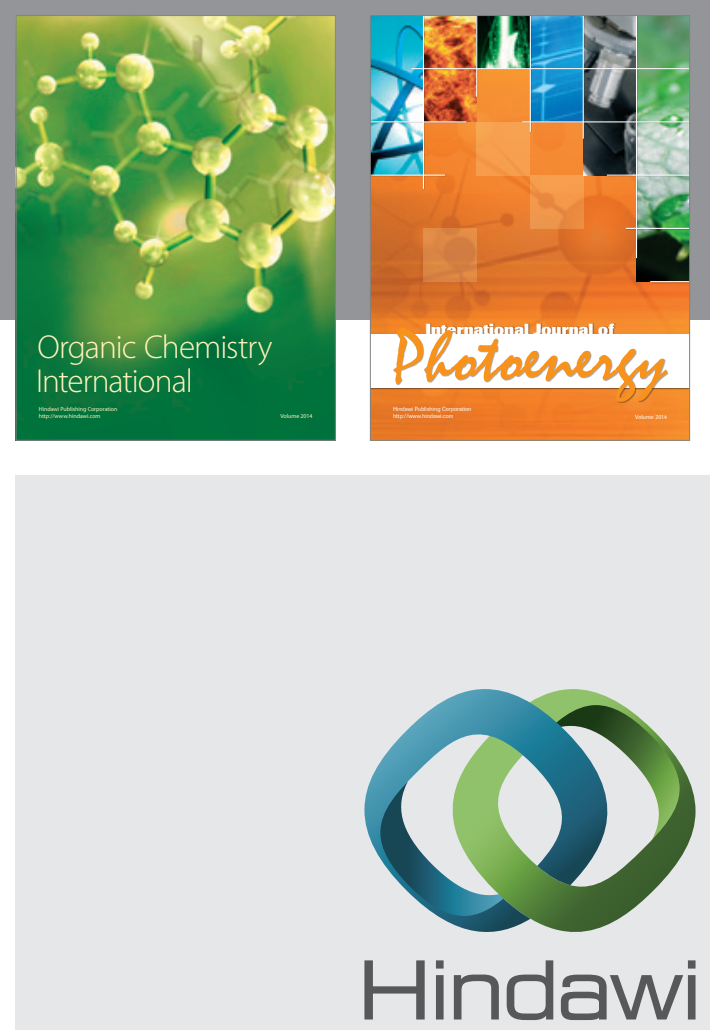

Submit your manuscripts at

http://www.hindawi.com
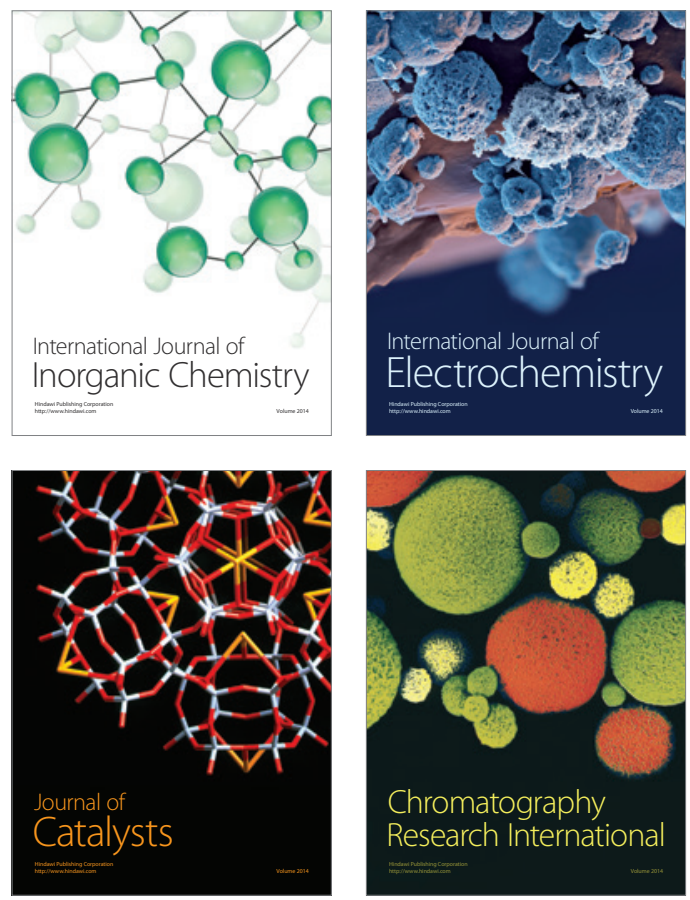
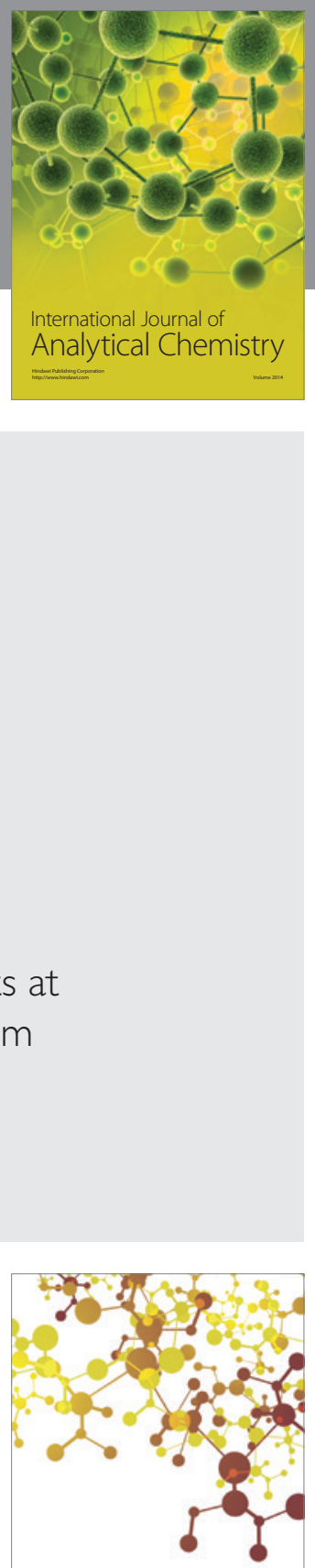

Journal of

Applied Chemistry
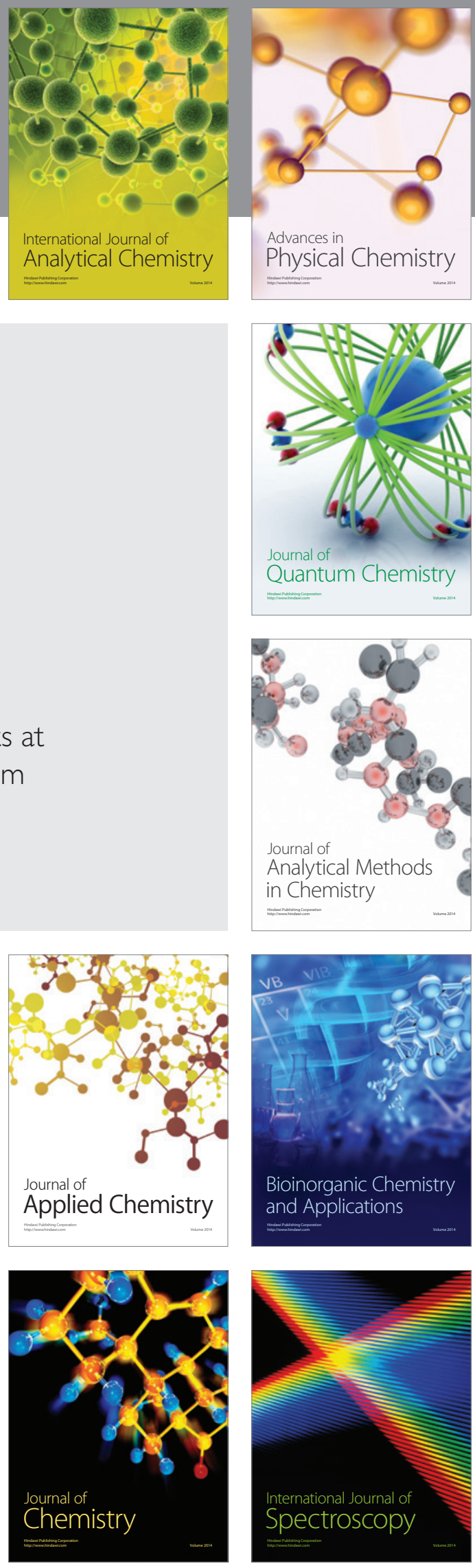